BULL. AUSTRAL. MATH. SOC.

VOL. $28(1983), 5-8$.

\title{
A BOUNDEDNESS CONDITION FOR SETS WITH LATTICE POINT CONSTRAINTS
}

\author{
P.R. SCOTT
}

Let $K$ be a closed convex body in $R^{n}$ containing a finite number of points of lattice $\Lambda$ in its interior. We show that $K$ is bounded if $K$ contains a certain suitably large simplex.

In his recent thesis, Arkinstall [1] has directed attention to convex bodies containing a finite number of interior lattice points. We give here a boundedness condition for such bodies.

Let $\Lambda$ be a lattice in the $n$-dimensional space $R^{n}$ having linearly independent generating vectors $v_{1}, v_{2}, \ldots, v_{n}$ from the origin 0. Let $K$ be a closed convex body in $R^{n}$ containing a finite number of points of $\Lambda$ in its interior. It is easy to see that $K$ may be chosen to extend beyond any preassigned bounds, even in $R^{2}$ (Figure 1 ).

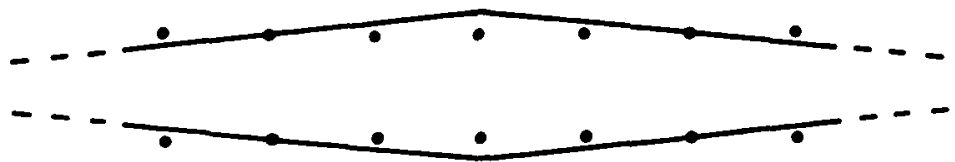

FIGURE 1

Received 1 March 1983. 
Denote by $\Delta_{n}$ the simplex in $R^{n}$ given by the convex hull of $v_{1}, v_{2}, \ldots, v_{n}$, and let $k \Delta_{n}$ represent any translate of the simplex obtained from $\Delta_{n}$ by enlargement about 0 with scale factor $k(>0)$.

LEMMA. If $k>n$, then $k \Delta_{n}$ contains a point of $\Lambda$ in its interior.

Proof. Since the statement of the lemma is invariant under affine transformation, we may take $\Lambda$ to be the integer lattice, and $v_{1}, v_{2}, \ldots, v_{n}$ the unit vectors $e_{1}, e_{2}, \ldots, e_{n}$ respectively. In the same way, we may now assume that a face of $k \Delta_{n}$ lies in' the hyperplane $x_{n}=\delta \quad(-1 \leq \delta<0)$.

We give a proof by induction on the dimension $n$. The result is trivially true for $n=1$. Let us assume it to be true for $n-1$, and consider $k \Delta_{n}$ with $k>n$. By simple proportion, the hyperplane $x_{n}=0$ now cuts $k \Delta_{n}$ in an $(n-1)$-dimensional simplex $q \Delta_{n-1}$, where

$$
q>k \cdot(k-1) / k=k-1>n-1 \text {. }
$$

Hence this section of $k \Delta_{n}$ contains a point of $\Lambda$ in its relative interior; this point is interior to $k \Delta_{n}$. This completes the proof by induction.

We now come to our main result.

THEOREM. Let $K$ be a closed convex body in $k^{n}$ containing a finite number of points of lattice $\Lambda$ in its interior. If $K$ contains a simplex $q \Delta_{n}$ with $q>n-1$, then $K$ is bounded.

Proof. As before, we take $\Lambda$ to be the integer lattice generated by the unit vectors, and we assume that a face of $q \Delta_{n}$ lies in each of the hyperplanes $x_{j}=\delta_{j} \quad\left(-1 \leq \delta_{j}<0,1 \leq j \leq n\right)$.

Since $K$ contains a finite number of lattice points in its interior, for each integer $j, l \leq j \leq n$, there exists an integer $B_{j}>0$ such that these lattice points lie in the strip $\left|x_{j}\right|<B_{j}$. We assert now that 
for each $j, 1 \leq j \leq n$, the body $K$ itself lies in the strip

$$
\left|x_{j}\right| \leq \frac{q B_{j}+(n-1)}{q-(n-1)}=B_{j}^{*} \text { say. }
$$

There are three cases to consider.

CASE 1. The bound $x_{j} \leq B_{j}^{*}$. Suppose $x^{*}$ is a point of $K$ lying in the region $x_{j}>B_{j}^{*}$. By convexity, $K$ contains the simplex $S$ which is the convex hull of $x^{*}$ and the face of $q \Delta_{n}$ lying in the hyperplane $x_{j}=-\delta_{j} \quad\left(0<\delta_{j} \leq 1\right)$.

Using simple proportion we see that the hyperplane $x_{j}=B_{j}$ cuts the simplex $S$ in an (n-1)-dimensional section $q^{\prime} \Delta_{n-1}$, where

$$
\begin{aligned}
q^{\prime} & >q\left(B_{j}^{*}-B_{j}\right) /\left(B_{j}^{*+\delta_{j}}\right) \\
& \geq q\left(B_{j}^{*}-B_{j}\right) /\left(B_{j}^{*}+1\right) \\
& =n-1,
\end{aligned}
$$

substituting for $B_{j}^{*}$ from (1).

Now by the lemma, $q^{\prime} \Delta_{n-1}$ contains a lattice point in its relative interior. This lattice point is interior to $S$ and so to $K$. But this contradicts our choice of $B_{j}$. Hence $K$ lies in the halfspace $x_{j} \leq B_{j}^{*}$.

CASE 2. The bound $x_{j} \geq-B_{j}^{*}$, but with either $\delta_{j}<1$ or $B_{j}>1$. (These alternative conditions on $\delta_{j}$ and ${ }_{j}{ }_{j}$ ensure that the hyperplane $x_{j}=-B_{j}$ does not intersect the simplex $q \Delta_{n} \cdot$ ?

Suppose $x^{*}$ is a point of $K$ lying in the half space $x_{j}<-B_{j}^{*}$, and construct $S$ as in Case 1. Now the hyperplane $x_{j}=-B_{j}$ intersects $S$ in an (n-1)-dimensional simplex $q^{\prime} \Delta_{n-1}$, where

$$
\begin{aligned}
q^{\prime} & >q\left(B_{j}^{*-B} j\right) /\left(B_{j}^{*}-\delta_{j}\right) \\
& >q\left(B_{j}^{*-B}\right) /\left(B_{j}^{*}+1\right) \\
& =n-1,
\end{aligned}
$$

and the proof follows as for case 1. 
CASE 3. The bound $x_{j} \geq-B_{j}^{*}$, and $\delta=1=B_{j}$. We show here that in fact $K$ lies in the halespace $x_{j}>-B_{j}\left(\geq-B_{j}^{*}\right)$.

Since $\delta=1$, the hyperplane $x_{j}=-1$ meets $q \Delta_{n}$ in a simplex $q \Delta_{n-1}$, where $q>n>n-1$. By the lemma, $q \Delta_{n-1}$ contains a lattice point $b$ in its relative interior. Since $B_{j}=1, b$ is not interior to $K$; hence $\mathbf{b}$ is a boundary point of $K$. Since $K$ is convex, there exists a support hyperplane $H$ to $K$ (and so to $q \Delta_{n}$ ) at b. It follows that $B$ must be the hyperplane $x_{j}=-B_{j}$. Thus $K$ lies in the halfspace $x_{j} \geq-B_{j} \geq-B_{j}^{*}$ as required.

This completes the proof of the theorem.

We define a lattice simplex to be a simplex which has every vertex at a lattice point. We then have the somewhat weak but interesting condition for the boundedness of $K$.

COROLLARY. If $K$ contains a lattice simplex $n \Delta_{n}$ then $K$ is bounded.

Finally, we observe that the two-sided bound $B_{j}^{*}$ given by (I) cannot be improved. For take $K$ to be the triangle in the plane with vertices $(-1,1),(-1,-1)$ and $(3,-1)$. Now $K$ contains the origin as its single interior lattice point, and $K$ contains the triangle $2 \Delta_{2}$. Hence $n=2, q=2, B_{1}=1$, and the bound $B_{1}^{*}=3$, obtained from the formula (I) is actually attained by $K$.

\section{Reference}

[1] John Robert Arkinstall, "Generalisations of Minkowski's theorem in the plane" (PhD thesis, University of Adelaide, Adelaide, 1982).

Department of Pure Mathematics, University of Adelaide, GPO Box 498, Adelaide, South Australia 5001, Australia. 